\section{SAT0011 MERTK+ MONOCYTES ARE EXPANDED IN THE PERIPHERAL BLOOD OF PATIENTS WITH ACTIVE IGG4- RELATED DISEASE AND INFILTRATE AFFECTED ORGANS}

L. Rovati ${ }^{1,2}$, E. Della Torre ${ }^{1,2}$, C. Sciorati ${ }^{1}$, E. Rigamonti ${ }^{1}$, M. Lanzillotta ${ }^{1}$, E. Bozzalla Cassione ${ }^{1}$, E. Bozzolo ${ }^{1}$, L. Dagna ${ }^{1}$, A. Manfredi'. ${ }^{1}$ Unit of Immunology, Rheumatology, Allergy and Rare Diseases, San Raffaele Scientific Institute and Università Vita-Salute San Raffaele, Milan, Italy; ${ }^{2}$ Ragon Institute of MGH, MIT and Harvard, Cambridge, MA, USA

Background: IgG4-Related Disease (IgG4-RD) is a multi-organ fibro-inflammatory disorder characterised by tumefactive lesions with well-defined histological features including lymphoplasmacitic infiltrate rich in lgG $4^{+}$plasma cells, storiform fibrosis and obliterative phlebitis ${ }^{1}$. Alternatively-activated macrophages (M2) have also been reported to abundantly infiltrate IgG4-RD lesions, but their role in IgG4-RD pathogenesis remains elusive. M2 macrophages have been recently shown to modulate innate and adaptive immune responses as well as tissue fibrosis by direct interaction with stromal cells ${ }^{2-4}$. Both these roles can be mediated by Mer tyrosine kinase (MerTK), a member of the TAM - Tyro3, Axl and MerTKreceptor family, which is highly expressed on M2 macrophages 5 . The relevance of MerTK and of its ligands Protein S (ProS) and Growth arrest-specific protein 6 (Gas6) in IgG4-RD has never been assessed before.

Objectives: To assess a pathogenetic relevance of the MerTK-ProS/Gas6 axis in $\lg$ G4-RD.

Methods: Immunohistochemical studies for CD68, CD163 and MerTK were performed on 8 cases of IgG4-RD involving different organs. MerTK expression within the different circulating monocyte subsets was quantified by flow cytometry both in 11 active untreated IgG4-RD patients and in 10 healthy controls (HC). Plasma levels of ProS, Gas6 and of their decoy receptor - soluble Mer (sMer) were measured by ELISA in $34 \mathrm{IgG} 4-\mathrm{RD}$ patients and $20 \mathrm{HC}$.

Results: MerTK was abundantly expressed in IgG4-RD lesions both within the inflammatory infiltrate and in the newly formed fibrous tissue. The pattern of MerTK expression was similar to that of the M2 macrophage marker CD163 (figure 1). Total circulating monocytes and their subsets were not expanded in active untreated IgG4-RD compared to HC. However, MerTK ${ }^{+}$monocytes were significantly increased in the peripheral blood of patients with lgG4-RD compared to $\mathrm{HC}$ $(p<0.001)$. Plasma levels of Gas6 and ProS were significantly higher in IgG4-RD patients compared to $\mathrm{HC}(\mathrm{p}=0.003$ and $\mathrm{p}=0.01$, respectively), while the plasma level of sMer was comparable to that of $\mathrm{HC}$. The number of $\mathrm{MerTK}^{+}$monocytes correlated positively with the number of circulating plasmablasts $(r=0.6 ; p<0.05)$ and negatively with Gas6 plasmatic concentration $(r=-0.7 ; p<0.05)$.
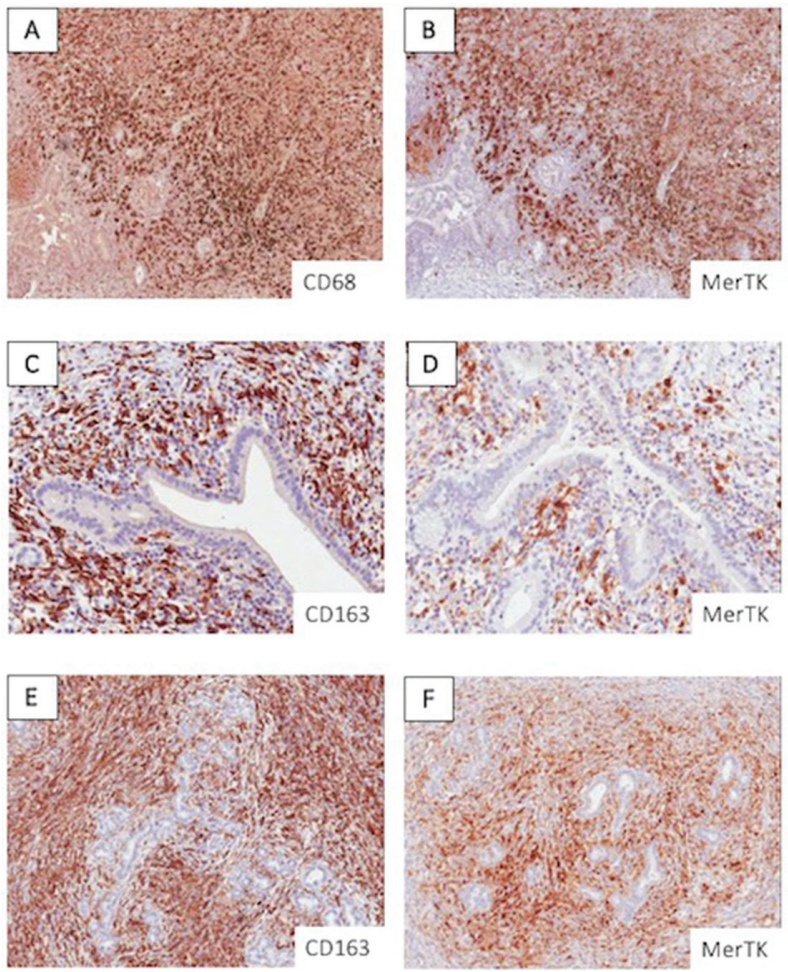

Abstract SAT0011 - Figure 1. Distribution of macrophages and MerTK-expressing cells in the lung (A-B), pancreas (C-D) and biliary tree (E-F) of IgG4-RD patients. Each set of sections was stained with MerTK, CD68 as a marker of both M1 and M2 macrophages and CD163 as a marker of M2 macrophages.
Conclusions: A subset of MerTK ${ }^{+} \mathrm{M} 2$ macrophages abundantly infiltrates IgG4 $\mathrm{RD}$ fibrotic lesions and their $\mathrm{MerTK}^{+}$monocyte precursors are expanded in the peripheral blood of patients with active IgG4-RD. MerTK ligands are also increased in IgG4-RD, suggesting an augmented activation of MerTK signalling pathways. Further studies are needed to better characterise this monocyte/mac rophage subpopulation, to understand its role in IgG4-RD and to identify possible biomarkers and therapeutic targets.

\section{REFERENCES :}

[1] Deshpande V, et al. Mod. Pathol 2012

[2] Furukawa S, et al. Clin. Immunol 2015.

[3] Furukawa S, et al. Sci. Rep 2017.

[4] Ohta M. et al. Med 2016.

[5] Rothlin CV, et al. Annu. Rev. Immunol 2015.

Disclosure of Interest: None declared

DOI: 10.1136/annrheumdis-2018-eular.5748

\section{SAT0012 \\ C-REACTIVE PROTEIN: NOT ONLY A MARKER, BUT ALSO A CAUSE OF INFLAMMATION THROUGH METABOLIC REPROGRAMMING OF HUMAN MACROPHAGES}

M. Newling ${ }^{1}$, L. Sritharan ${ }^{1}$, B. Everts ${ }^{2}$, L. de Boer ${ }^{3}$, S. Zaat ${ }^{3}$, D. Baeten ${ }^{1}$, J. den Dunnen $1 .{ }^{1}$ Clinical Immunology and Rheumatology, Academic Medical Center (AMC), Amsterdam; ${ }^{2}$ Parasitology, Leiden University Medical Center (LUMC), Leiden; ${ }^{3}$ Medical Microbiology, Academic Medical Center (AMC), Amsterdam, Netherlands

Background: C-reactive protein (CRP) is an acute-phase protein produced in high quantities by the liver in response to infection and during chronic inflammatory disorders such as rheumatoid arthritis (RA). As a consequence, CRP is in widespread clinical use as a general marker of inflammation. Although CRP is known to facilitate clearance of cell debris by phagocytic cells by binding to its ligand phosphocholine on dead cells, additional functions of CRP are still not completely understood.

Objectives: Here, we set out to investigate whether CRP, which is present in high concentrations in synovial fluid of active RA patients, also plays a role in the orchestration of inflammation in the inflamed joint.

Methods: Human macrophages were differentiated from blood monocytes of healthy volunteers. Cells were stimulated with complexed CRP (c-CRP) and/or ligands for Toll-like receptors (TLRs), mimicking the stimuli in the inflamed joint. Responsible signalling pathways were identified using small molecule inhibitors and RNA interference. Metabolic pathways were identified using specific inhibitors and the Seahorse metabolic analyzer.

Results: Strikingly, we here provide evidence that CRP is not only a marker, but also a cause of inflammation by strongly amplifying the production of RA-associated pro-inflammatory cytokines. We show that complex formation of CRP as a result of binding to its ligand phosphocholine selectively enhanced TNF $\alpha$, IL-1 $\beta$, and IL-23 production by human macrophages. While c-CRP did not induce cytokine production individually, C-CRP synergized with TLRs to amplify cytokine gene translation. We identified Fc gamma receptor I and Ila (FcyRI and FcyRlla) as the main receptors responsible. Moreover, we unravelled the responsible molecular mechanism of c-CRP-induced inflammation, which crucially depends on signalling through kinases Syk and PI3K, resulting in enhanced gene translation of pro-inflammatory cytokines through metabolic reprogramming, particularly through amplified glycolysis and fatty acid synthesis.

Conclusions: These data indicate that CRP is not only a marker, but might also be a cause of inflammation in RA patients by selectively promoting RA-associated pro-inflammatory cytokine production by human macrophages, thereby exacerbating pathology. From a therapeutic point of view, inhibition of c-CRP-induced immune activation, e.g. by targeting the identified molecular mechanisms, may be a valuable tool to suppress inflammation.

Disclosure of Interest: M. Newling: None declared, L. Sritharan: None declared, B. Everts: None declared, L. de Boer: None declared, S. Zaat: None declared, D. Baeten Employee of: Union Chimique Belge, J. den Dunnen: None declared DOI: 10.1136/annrheumdis-2018-eular.5107 